\section{Watching single lipids move}

Multiple photodiode detectors are used to track the transit of dye-labeled single lipids through an excitation spot at high resolution.

One of the challenges of working at the cutting edge of methodological development is that when a new method gives insight into a process that has not been previously measurable, it is far from trivial to validate the results with an independent approach. This is a challenge that will not be unfamiliar to many of the readers of Nature Methods.

When Christian Eggeling, Stefan Hell and colleagues at the Max Planck Institute, Goettingen combined stimulated emission depletion (STED) microscopy with fluorescence correlation spectroscopy (FCS) and observed last year in a paper in Nature that certain lipids are trapped in small ( $<20$-nanometer) domains in the plasma membrane of living cells, there remained some questions about whether this striking result could be obtained with other methods. Or did the particularities of the STED measurement perhaps influence the results in some way? So Hell and colleagues went about developing an alternative approach to watching lipids diffuse at the surface of live cells.

"We estimated that it should be possible to see the trajectories of individual labeled molecules with a microscope that is very much akin to a confocal microscope but has three detectors, just slightly offset, covering the excitation volume," explains Hell. As a labeled lipid passes through the excitation volume, it is detected by all three avalanche photodiode detectors, and its position in the focal area can be very precisely localized. Notably, this tracking must be carried out at a 100 -fold lower concentration of labeled lipid than has been used for STED-FCS, and without the use of a STED beam, yet it yields very similar results to what the researchers had previously seen using STED-FCS. A sphingolipid, unlike a phosphoglycerolipid, is trapped on a time scale of $\sim 15$ milliseconds in membrane domains less than 20 nanometers in diameter, in a cholesterol-dependent manner.

In contrast to camera-based single-molecule lipid tracking, the detector-based approach permits tuning of the spatial and temporal resolution by adjusting the time window over which photons are binned. It suffers from a relatively small field of view - a spot of 240 nanometers in diameter in the reported experiments - but for the examination of macromolecular complexes, or events occurring on the scale of tens of nanometers, this is sufficient. "It was very pleasing to us," says Eggeling, "that we obtained the same results with completely orthogonal methods. We put both methods on a more solid ground."

\section{Natalie de Souza}

\section{RESEARCH PAPERS}

Sahl, S.J. et al. Fast molecular tracking maps nanoscale dynamics of plasma membrane lipids. Proc. Natl. Acad. Sci. USA 107, 6829-6834 (2010). 